\title{
La cobertura arbórea de Acacia caven sobre la calidad de la pradera y microclima en un sistema silvopastoril de Chile central
}

\author{
Acacia caven cover on the pasture quality and microclimate in a \\ silvopastoral system of central Chile
}

\author{
Alejandro Lucero Ignamarca1,2*, Fernando Muñoz Sáez², Jorge Cancino Cancino², Alvaro Sotomayor Garretón!, \\ Francis Dube², Katia Sáez Carrillo³, Arnoldo Villarroel Muñoz’ y Mauricio Navarrete Torres' \\ Instituto Forestal (INFOR), Sede Biobío. Concepción, \\ Chile. \\ 2 Universidad de Concepción. Facultad de Ciencias \\ Forestales. Concepción, Chile. \\ ${ }^{3}$ Universidad de Concepción. Facultad de Física y \\ Matemáticas. Departamento de Estadísticas. \\ Concepción, Chile.
}

\section{RESUMEN}

El presente estudio tiene como objetivo determinar el efecto de diferentes coberturas arbóreas sobre productividad y calidad de la pradera y sobre variables microclimáticas, en un sistema silvopastoril con espino (Acacia caven). Para ello se estableció un ensayo con cuatro tratamientos de cobertura arbórea ( $0 \%, 30 \%-40 \%, 50 \%-60 \%,>70 \%$ ), en un diseño experimental de bloques aleatorizados completos y tres repeticiones. Además de variables relacionadas con la productividad y calidad de la pradera, mediante sensores se realizó la medición permanente de temperatura y humedad de suelo, temperatura ambiente y humedad relativa. El periodo de análisis incluye dos temporadas de crecimiento de la pradera. Para los análisis estadísticos se consideraron tres modelos; i) la cobertura arbórea sobre la productividad de la pradera; ii) la cobertura arbórea sobre las variables microclimáticas y iii) la cobertura arbórea sobre la productividad pratense, considerando las variables microclimáticas como covariables. Los resultados indican que, en la segunda temporada, la mayor productividad se alcanzó con el tratamiento sin presencia de árboles $(0 \%)$. No se detectó efecto de la cobertura arbórea sobre la calidad de la pradera, ni sobre variables microclimáticas, para los periodos evaluados. Las variables microclimáticas que mejor explicaron el desarrollo de la pradera fueron la humedad relativa mínima y la temperatura ambiente. Transcurridas dos temporadas de crecimiento, no es factible dilucidar aún cuál tratamiento genera las mejores condiciones para la productividad de la pradera, por lo que se hace necesario continuar con las mediciones.

PALABRAS CLAVE: agroforestería, espino, estepa de Acacia caven, sistema silvopastoril, valor pastoral, variables microclimáticas.

\section{ABSTRACT}

The objective of this study was to determine the effect of different tree covers on the productivity and quality of the pasture and on specific microclimatic variables in an Acacia caven-based silvopastoral system. A trial that included four tree-cover treatments $(0 \%, 30 \%$ $40 \%, 50 \%-60 \%$ and over 70\%) was established in an experimental set-up with complete randomized blocks and three repetitions. In addition to the productivity and quality of the pasture, probes were used for the continuous measurement of soil temperature and moisture at $15 \mathrm{~cm}$ depth, ambient temperature and relative humidity. The period of analysis included two prairie growth seasons. Three models were considered for the statistical analyzes: i) tree cover on pasture productivity; ii) tree cover on microclimatic variables; and iii) the prairie productivity, considering the microclimatic variables as covariables. The results indicate that in the second growing season, the highest productivity was achieved with the tree-less treatment $(0 \%$ cover $)$. No effect of tree cover on the quality of the prairie was detected, nor on the microclimatic variables in either period. The microclimatic variables that best explained the development of the prairie were the minimum relative humidity and the ambient temperature. After two growing seasons, it is not possible to determine which treatment generates the best conditions for the productivity of the pasture, so it is necessary to continue with the measurements.

KEYWORDS: Agroforestry, Espino, steppe of Acacia caven, silvopastoral system, pastoral value, microclimatic variables. 


\section{INTRODUCCIÓN}

Los sistemas silvopastoriles son los más utilizados en distintas zonas geográficas del mundo, incluyendo Chile. Particularmente, la estepa de Acacia caven (Mol.) Mol (espinal) presenta un importante potencial para ser usado bajo este tipo de sistemas; este uso permite darle mayor sustentabilidad al recurso (Ovalle, 1986), dado que históricamente ha tenido una fuerte intervención antrópica que lo ha degradado (Olivares, 2006).

El espinal está presente en la zona central de Chile, abarcando una superficie aproximada de 950000 hectáreas ${ }^{1}$, desde la Región de Coquimbo (paralelo $30^{\circ} 00^{\prime} 00^{\prime \prime}$ S) hasta la del Biobío (paralelo 38 $38^{\circ} 00^{\prime} 00^{\prime \prime}$ S). Esta formación tiene como especie leñosa principal a Acacia caven (espino), que es un árbol de $2 \mathrm{~m}$ a $6 \mathrm{~m}$ de altura, es la única especie del género que crece naturalmente en Chile (Cabello y Donoso, 2006) y es clasificada dentro del tipo forestal esclerófilo, subtipo espinal.

En los sistemas silvopastoriles con Acacia caven, la presencia o ausencia de árboles altera la composición, distribución espacial, calidad pastoral y productividad de las especies que componen la pradera presente en ellos (Ovalle y Squella, 1988). El efecto de los árboles sobre la pradera depende de varios factores y de cómo estos se interrelacionan. Scholes y Archer (1997) mencionan cuatro conjuntos de factores, 1) las características ecofisiológicas o los hábitos de crecimiento de los árboles y las hierbas (arquitectura de la copa y las hojas, sistemas radiculares, entre otros); la vía fotosintética utilizada (plantas C3, C4 y CAM); el hábito fotosintético (árboles de hoja perenne o caduca); y los requerimientos específicos de los recursos existentes (luz, agua, nutrientes); 2) la disponibilidad de recursos hídricos (precipitaciones) y edáficos; 3) la existencia de sistemas de pastoreo extensivo y depredación de semillas; 4) la frecuencia, la intensidad y la zona de impacto de perturbaciones ambientales (por ejemplo: incendios).

1 Superficie estimada por el Área de Inventario y Monitoreo de Ecosistemas Forestales, Línea de Inventario Forestal Continuo. Instituto Forestal Sede Biobío, con base en información actualizada del Catastro
En un estudio sobre el comportamiento de la producción de materia seca de la pradera, que consideraba áreas con y sin influencia directa del espino y con diferentes distanciamientos entre copas de los árboles, se pudo comprobar que la cantidad de materia seca de la pradera siempre fue mayor bajo la proyección de copa, fluctuando entre $2900 \mathrm{~kg} \mathrm{ha}^{-1}$ y $3575 \mathrm{~kg} \mathrm{ha}^{-1}$ y, en las áreas sin influencia, disminuyó a $1000 \mathrm{~kg} \mathrm{ha-1}$ (Olivares, 2006). Ovalle y Avendaño (1984a), estudiaron los cambios en producción de forraje en relación con diferentes porcentajes de la cobertura arbórea de espino (30\%, 50\% y $80 \%$ ). Como resultado, se observó una relación directa entre producción de materia seca (MS) y cobertura arbórea, con valores de $2780 \mathrm{~kg}, 3274 \mathrm{~kg}$ y $3966 \mathrm{~kg}$ de materia seca por hectárea por año, para 30\%, 50\% y 80\% de cobertura, respectivamente. Esto es ratificado por Fernández, Squella y Ovalle (2002), quienes indican que el mantener una cobertura de espinos implica mayor producción y calidad de forraje.

En cuanto a la composición botánica de la pradera, se ha observado que esta cambia por efecto del árbol, porque, al aumentar la cobertura arbórea, se incrementa la presencia de gramíneas forrajeras, en detrimento de las especies leguminosas menos forrajeras presentes en la pradera, aumentando por ello su valor pastoral (VP) (Ovalle y Avendaño, 1984a; Ovalle, 1986; Olivares, 2006).

El hecho de que la cobertura de copa ejerza efecto positivo sobre el crecimiento y composición botánica de la pradera que crece bajo su influencia es producto de los mejores niveles de materia orgánica, nitrógeno (N) y humedad disponible en el suelo. Fuera de la proyección de copa, en general, se presentan condiciones con mayor temperatura, menores niveles nutritivos y de humedad, provocando un menor crecimiento de la pradera (Olivares, Cornejo y Gándara, 1983; Ovalle y Avendaño, 1984b).

$A$. caven crea condiciones microambientales que posibilitan una mejor y mayor disponibilidad de materia

de los Recursos Vegetacionales Nativos de Chile (al año 2015) de la Corporación Nacional Forestal (Conaf). 
seca en la pradera (Olivares, 2006). Esto es producto de que disminuye la radiación incidente a nivel del estrato herbáceo, atenuando el microclima térmico, mejorando los balances hídrico y trófico, conjunto de condiciones que determinan cambios importantes en el funcionamiento del estrato herbáceo (Ovalle, 1986).

A nivel del suelo, los árboles generan condiciones que permiten una mayor disponibilidad hídrica para las plantas, producto de la reducción de la temperatura del suelo y el aumento de la humedad relativa del aire, lo que provoca una disminución en la evapotranspiración de las plantas. El balance final es que las áreas arboladas son más húmedas que las no arboladas, favoreciendo la producción de materia seca de la vegetación que crece bajo su influencia (Ovalle y Avendaño, 1984b).

\section{OBJETIVOS}

Este estudio tuvo como objetivo determinar, en un sistema silvopastoril asociado a la estepa de Acacia caven (Mol.) Mol, el efecto que tienen coberturas arbóreas de $0 \%, 30 \%$ - 40\%, $50 \%-60 \%$ y $>70 \%$ sobre la productividad y calidad forrajera de la pradera y sobre las variables microclimáticas humedad y temperatura del suelo, temperatura ambiente y humedad relativa, antecedentes que permitirán aportar al manejo sustentable de este recurso. En este sentido se plantea la hipótesis de que la modificación de la cobertura arbórea por medio de la corta selectiva de árboles o vástagos de espino bajo un esquema de producción silvopastoril, permite aumentar la cantidad y mejorar la calidad de la pradera natural en comparación con la pradera que se desarrolla en sectores donde se eliminan totalmente los espinos.

\section{MATERIALES Y MÉTODOS}

Ubicación. El área de estudio cubre una superficie de 2.4 ha de espinal adulto, coordenadas lat. $-35.892717^{\circ}$ long. $72.145670^{\circ}$, localizada en el Predio "San Adolfo" Hijuela 5 Sector Caliboro km 48 de la Ruta "Los Conquistadores", Comuna de Cauquenes, Región del Maule, zona central de Chile.
Clima. El clima de la Comuna de Cauquenes es del tipo mediterráneo, con precipitaciones concentradas en el período frío del año y con sequía durante la estación más cálida (Di Castri y Hayek, 1976). Sesenta por ciento de las precipitaciones se concentran durante los meses junio a agosto, con un promedio anual de $696 \mathrm{~mm}$ y un periodo seco de 7 meses. El régimen térmico se caracteriza por temperaturas que varían, en promedio, entre una máxima en enero de $29.0^{\circ} \mathrm{C}$ y una mínima en julio de $4.9^{\circ} \mathrm{C}$, con una temperatura media anual de $14.1^{\circ} \mathrm{C}$. El período libre de heladas es de 259 días, con un promedio de seis heladas por año. Este es un factor limitante para muchas especies frutales y de uso forestal. Dicho periodo se extiende desde mediados de octubre hasta mediados de abril. La menor influencia oceánica favorece una amplitud térmica mayor que las zonas vecinas, con inviernos relativamente fríos y veranos calurosos (Santibáñez y Uribe, 1993).

Suelo. El área de estudio se encuentra dentro de la Serie Cauquenes (CQ), de la familia fina, caolinítica, isomésica de los Ultic Palexeralfs (Alfisol) (Martínez, 2004). Son suelos profundos, formados "in situ” a partir de rocas graníticas, bien evolucionados, de texturas arcillosas en todo el perfil. A nivel freático es de permeabilidad muy lenta y escurrimiento superficial lento. En el caso específico de la zona de estudio, esta se encuentra dentro de la variación CQ-2 de esta serie, que corresponde a la fase casi plana con pendiente de $1 \%$ a $3 \%$, de textura superficial franco arcillosa. Suelo profundo (más de $100 \mathrm{~cm}$ ) y de drenaje moderado. Presenta nivel freático a $100 \mathrm{~cm}$ de profundidad hasta principios de primavera (Centro de Información de Recursos Naturales [Ciren], 1997).

Componente arbórea. La caracterización de esta componente en la zona evaluada se inició en junio del 2013, contempló la ejecución de un censo dentro de toda el área de estudio, donde cada árbol, mono y plurifustal, fue codificado con $\mathrm{N}^{\circ}$ de árbol, $\mathrm{N}^{\circ}$ de Vástago y $\mathrm{N}^{\circ}$ de subvástago, estos dos últimos para los individuos plurifustales. Al inicio, cada árbol o centro de cepa, para el caso de individuos plurifustales, se ubicó espacialmente dentro de cada parcela de $30 \mathrm{~m} \times 30 \mathrm{~m}$, a través de puntos cartesianos 
$(x, y)$, considerándose el punto de origen $(0,0)$ el vértice $\mathrm{NE}$ de cada parcela. Posteriormente, de cada árbol se le registraron las siguientes variables: especie, calidad (en relación a forma y sanidad), tipo (mono o plurifustal), radios de copa en ocho puntos cardinales (N, NE, E, SE, S, SO, $\mathrm{O}, \mathrm{NO}$ ), diámetro a la altura del cuello (DAC), diámetro a la altura del pecho (DAP) y altura total del fuste o de cada vástago, altura de inicio de copa por árbol o cepa.

Cobertura de copa. Esta actividad se realizó a través de la determinación de la longitud de la proyección perpendicular al suelo de la copa para cada árbol o cepa en ocho puntos cardinales a través de la observación directa en terreno. Posteriormente se transformaron estos radios vectores en coordenadas $(\mathrm{x}, \mathrm{y})$ en cada unidad experimental (UE). Esta información fue sistematizada y exportada al ambiente ArcMap 9.3, el cual a través de herramientas de análisis y superposición de capas (overlay), determinó los perfiles horizontales y calculó la cobertura para cada UE antes de la aplicación de los tratamientos.

Componente pratense. Para caracterizar la pradera en cuanto a su materia seca, composición botánica y valor pastoral, se establecieron tres jaulas al interior de cada tratamiento y sus respectivas repeticiones, de superficie conocida de $0.5 \mathrm{~m}^{2}(50 \mathrm{~cm} \times 100 \mathrm{~cm})$, las cuales fueron fijadas al suelo (Ovalle y Avendaño, 1984a), para posteriormente cosechar desde cada una de ellas la pradera para sus análisis. Estas jaulas se instalaron en octubre del 2014, aunque la zona de estudio se encontraba en exclusión de ganado desde que se aplicaron los tratamientos de cobertura respectivos, por lo que no se vio afectado el crecimiento inicial de la pradera por efecto de ganado. La pradera fue cosechada con tijera durante dos temporadas, septiembre a diciembre del 2014 y septiembre del 2015 a febrero del 2016. Las muestras fueron enviadas para su clasificación al laboratorio de la Estación Experimental de Cauquenes perteneciente al Instituto de Investigaciones Agropecuarias (INIA).

Para la determinación de la productividad de la pradera (PP), en primer lugar, se hizo la clasificación de las distintas especies herbáceas componentes de la muestra. Cada submuestra por especie fue secada separadamente en horno de ventilación forzada a $80{ }^{\circ} \mathrm{C}$ por 48 horas y, posteriormente, pesada con una pesa digital marca Radwag modelo WTB 2000 (USA), determinando el peso seco por especie, el peso total por muestra y por tratamiento para cada bloque (Ovalle, Avendaño, Etienne, Muñoz y Serra, 1981). El peso total, es decir la suma de las submuestras por especies para cada tratamiento, bloque y mes, fue extrapolado a la hectárea para determinar el peso seco total generado en cada situación, expresado en kilogramos de masa seca por hectárea, a través de un factor de expansión, de acuerdo con la ecuación 1 .

$$
P P_{i j k}=P S_{i j k} * K \quad[1]
$$

Donde:

$P P_{i j k}=$ Productividad de la pradera como peso seco total tratamiento "ip" bloque “j” y mes " $k$ " (kg ha-1)

$P S_{i j k}=$ Peso seco total de la muestra del tratamiento " $\mathrm{i}$ " Bloque “j” y mes " $k$ " (kg)

$K=\quad$ Factor de expansión a la hectárea

Para la determinación del valor pastoral de la pradera (VP), inicialmente se requirió caracterizar la pradera en cuanto a su composición botánica, por lo cual se clasificaron para cada muestra, las distintas especies componentes. Esta situación se hizo más compleja al corresponder a muestras mensuales, dado que las características diferenciadoras de las especies (flores, semillas, u otros) no estaban presentes en todas ellas; esto no sucede cuando se analiza una sola muestra para toda la temporada. Una vez clasificadas, cada muestra fue secada y pesada en laboratorio. La metodología para calcular VP consistió en determinar un índice de calidad, con base en las diferentes proporciones de las especies presentes en la pradera (composición botánica), dado que cada especie tiene valores nutrimentales específicos. Este valor nutritivo es representado por un índice específico (IE), que considera aspectos tales como palatabilidad, valor nutritivo y recuperación al corte, entre otros. Este índice es un valor que fluctúa entre 0 y 10 y está 
basado en la determinación hecha por el Centre d' Etudes Phytosociologiques et Êcologiques (CEPE) para las especies de la pradera mediterránea subhúmeda de Francia; con ese mismo índice se calificaron las especies nativas y naturalizadas de las praderas de Chile mediterráneo central por Ovalle et al. (1981). El VP de las muestras de pradera se calcula utilizando la ecuación 2.

$$
V P_{i j k}=\sum\left(\left(\frac{P S_{i j k l}}{P S T_{i j k}}\right) * \mathrm{IE}_{l}\right)
$$

Donde:

$$
\begin{aligned}
& V P_{i j k}=\text { Valor pastoral del tratamiento “i” Bloque “j” } \\
& P S_{i j k l}=\text { Peso seco tratamiento “i”" Bloque “ } \mathrm{j} \text { ” muestra " } \mathrm{k} \text { ” } \\
& \text { especie "l" } \\
& P S T_{i j k}=\text { Peso seco tratamiento "i” Bloque “ } \mathrm{j} \text { ” muestra " } \mathrm{k} \text { ” } \\
& \mathrm{IE}_{l}=\text { Índice específico especie "l". Valor que fluctúa } \\
& \text { entre } 0 \text { y } 10
\end{aligned}
$$

Variables microclimáticas. Para el registro de las variables microclimáticas, se estableció una red de sensores permanentes para: temperatura y humedad del suelo a 15 $\mathrm{cm}$ de profundidad, temperatura ambiental y humedad relativa a 1.3 metros de altura, de acuerdo con la Norma de la Organización Mundial de Meteorología. Esta red de sensores se ubicó dentro de una protección contra la radiación directa y sobre un soporte, que mantuvo un flujo de aire adecuado para permitir que el sensor entrara en equilibrio con la temperatura del aire (Castro, 2008).

Considerado lo anterior, se instalaron para cada tratamiento y repeticiones, un set que incluyó un sensor de temperatura y humedad del suelo modelo 5TM y sensor de humedad relativa y temperatura ambiente con abrigo meteorológico modelo VP-3 RH, ambos de marca Decagon Devices (Washington, EU). Todos estos sensores fueron conectados a registradores de datos análogos (datalogger) de cinco puertos modelo Em50 marca Decagon

\footnotetext{
2 ShadeMotion es un software construido para modelar las sombras que proyectan los árboles sobre un terreno plano, horizontal o inclinado. El programa fue pensado como una herramienta de cálculo para investigadores en agroforestería, ciencias forestales, ganadería y otras áreas de la agricultura, en donde el conocimiento de la trayectoria y
}

Devices (Washington, EU), ubicados en terreno. Todos los sensores fueron programados para hacer el registro de variables en intervalos de una hora.

La decisión de la ubicación tanto de las jaulas como de los sensores consideró la sombra acumulada durante un año en el punto de fijación y aquel punto que acumulara un porcentaje de sombra dentro del mismo intervalo de cobertura aplicado para cada tratamiento, esto a través del Software ShadeMotion $3.0^{2}$ (Quesada, Somarriba y Malek, 2005).

Diseño experimental. Para determinar el efecto de la cobertura del espino sobre la cantidad y calidad de la pradera, se implementó un diseño de 3 bloques completamente aleatorizados (BCA) con cuatro tratamientos (Tabla 1), los cuales contemplaron modificaciones de la cobertura en tres niveles, más un testigo que no tuvo intervención. Se determinó este diseño en bloques para aislar la variabilidad probable, producto de distintas condiciones de humedad del suelo, por la presencia de un estero temporal a un costado del área de estudio. Para el establecimiento de los diferentes bloques y tratamientos, el área de estudio se orientó de norte a sur con una brújula Sunnto (Finlandia), generando un área total de 2.4 ha. Posteriormente, al interior de esta área se delimitaron sectores de $30 \mathrm{~m} \times 30 \mathrm{~m}$, generando una grilla de 22 parcelas orientadas también de norte a sur y dentro de la cual se ubican los bloques y las $12 \mathrm{UE}$ a evaluar, que correspondieron a los cuatro tratamientos distribuidos aleatoriamente dentro de cada uno de los bloques.

Sombra acumulada. De cada UE, se sistematizó e ingresó la información al software ShadeMotion 3.0 con las características dasométricas y ubicación espacial de todos los árboles existentes dentro de cada UE, el software permitió obtener una representación digital en 3D de cada una de estas UE y, posteriormente, realizar una simulación de sombra acumulada durante un año en toda la superficie

cantidad de sombra que proyectan un conjunto de árboles puede ser un factor importante a tomarse en cuenta. ShadeMotion permite calcular sombras en cualquier lugar del planeta y en cualquier intervalo de fechas y de horario (Quesada et al., 2005). 
TABLA 1. Tratamientos de cobertura arbórea

\begin{tabular}{cc}
\hline Tratamiento & $\begin{array}{c}\text { Cobertura promedio } \\
(\%)\end{array}$ \\
\hline T1 & 0 \\
T2 & $30-40$ \\
T3 & $50-60$ \\
T4 & $>70\left({ }^{*}\right)$ \\
\hline
\end{tabular}

(*) Situación testigo sin intervención

de las UE correspondiente a los tratamientos T2, T3 y T4 y sus repeticiones, generando una representación gráfica de sombras acumuladas para cada UE.

La aplicación de la herramienta ShadeMotion 3.0 permitió resolver una problemática planteada inicialmente por los autores, en relación con el real efecto de la sombra generada por la cobertura arbórea sobre la pradera en un sistema silvopastoril, dado que no solamente existe un efecto de la luminosidad de manera perpendicular a la copa de los árboles, sino que también existe un efecto lateral e incluso indirecto por el movimiento estacional diario del sol y lógicamente de la sombra que puede generarse en una temporada de crecimiento (Ovalle y Avendaño, 1984a; Olivares, 2006; Ramírez, 2011). Esta problemática y preocupación inicial se genera por el hecho de que la toma de datos de productividad de pradera, con jaulas de exclusión y de variables microclimáticas a través de sensores, es la representación de una medición puntual del lugar donde se obtiene la muestra de la pradera y se registra el dato microclimático, por lo tanto, la selección de ese punto debe necesariamente dar algún grado de certeza de que es representativo del tratamiento a evaluar.

Análisis estadístico. Se plantearon tres modelos. El primero tenía como objetivo modelar independientemente la productividad de la pradera (PP) y el valor pastoral (VP), en función de los diferentes tratamientos de cobertura. Para ello se hizo un análisis de varianza (Andeva), que permitió determinar si estos tratamientos afectaban de manera significativa o no a las variables respuesta PP y VP. La información resumida de los valores obtenidos para estas variables, se observan en las tablas 2 y 3 .

El segundo modelo analizó el efecto de los tratamientos sobre las diferentes variables microclimáticas, en este caso se ajustó un modelo de análisis de covarianza (Ancova), usando el tiempo como covariable y la cobertura arbórea como factor (Tabla 4). En las figuras 1, 2, 3 y 4 se puede apreciar el comportamiento de las distintas variables microclimáticas desde octubre de 2014 hasta septiembre de 2016 (2 años de captura de datos).

TABLA 2. Productividad de pradera por cobertura arbórea en dos temporadas.

\begin{tabular}{|c|c|c|c|c|c|c|c|c|c|c|c|c|c|}
\hline \multirow{2}{*}{ Temporada } & \multirow{2}{*}{ Mes } & \multicolumn{12}{|c|}{ Productividad de la Pradera $\left(\mathrm{kg} \mathrm{ha}^{-1}\right)$ x Cobertura Arbórea } \\
\hline & & \multicolumn{3}{|c|}{$T 1: 0 \%$} & \multicolumn{3}{|c|}{ T2: $30 \%-40 \%$} & \multicolumn{3}{|c|}{ T3: $50 \%-60 \%$} & \multicolumn{3}{|c|}{ T4: $>70 \%$} \\
\hline \multirow{5}{*}{2014} & 9 & 605.6 & \pm & 226.2 & 615.3 & \pm & 412.3 & 595.1 & \pm & 122.2 & 921.3 & \pm & 137.6 \\
\hline & 10 & 1054.2 & \pm & 146.7 & 555.3 & \pm & 52.5 & 534.2 & \pm & 74.7 & 446.4 & \pm & 148.2 \\
\hline & 11 & 464.0 & \pm & 164.0 & 383.3 & \pm & 84.4 & 335.6 & \pm & 18.2 & 145.6 & \pm & 47.3 \\
\hline & 12 & 154.9 & \pm & 29.4 & 219.8 & \pm & 44.3 & 118.0 & \pm & 8.5 & 97.8 & \pm & 39.6 \\
\hline & Total & 2278.7 & \pm & 96.5 b & 1773.8 & \pm & $491.6 \mathrm{~b}$ & 1582.9 & \pm & $158.1 \mathrm{~b}$ & 1611.1 & \pm & $\begin{array}{c}331.3 \\
\text { b }\end{array}$ \\
\hline \multirow{7}{*}{$2015-2016$} & 9 & 2097.1 & \pm & 1304.7 & 956.7 & \pm & 767.9 & $\begin{array}{c}1035 . \\
8\end{array}$ & \pm & 77.8 & 1014.2 & \pm & 445.5 \\
\hline & 10 & 562.4 & \pm & 145.3 & 357.3 & \pm & 32.4 & 308.4 & \pm & 43.1 & 219.3 & \pm & 60.5 \\
\hline & 11 & 743.3 & \pm & 53.2 & 421.1 & \pm & 62.0 & 403.8 & \pm & 89.1 & 236.7 & \pm & 109.1 \\
\hline & 12 & 120.7 & \pm & 89.2 & 242.9 & \pm & 35.0 & 161.3 & \pm & 87.3 & 88.7 & \pm & 27.1 \\
\hline & 1 & 41.3 & \pm & 13.8 & 93.3 & \pm & 66.0 & 74.2 & \pm & 61.7 & 36.4 & \pm & 7.3 \\
\hline & 2 & - & & - & 17.6 & \pm & 30.4 & 11.1 & \pm & 19.2 & - & & - \\
\hline & Total & 3564.9 & \pm & $\begin{array}{c}1098.4 \\
\text { a }\end{array}$ & $\begin{array}{c}2088 . \\
9\end{array}$ & \pm & 716.5 b & 1994.7 & \pm & $\begin{array}{c}237.1 \\
\text { b }\end{array}$ & 1595.3 & \pm & $\begin{array}{c}585.3 \\
\text { b }\end{array}$ \\
\hline
\end{tabular}

Nota: Letras iguales indican sin diferencia estadísticamente significativa $(p<0.05)$. 
TABLA 3. Valor pastoral (VP) de la pradera por cobertura arbórea en dos temporadas.

\begin{tabular}{|c|c|c|c|c|c|c|c|c|c|c|c|c|c|}
\hline \multirow{3}{*}{ Temporada } & \multirow{3}{*}{$\begin{array}{c}\text { Mes } \\
9\end{array}$} & \multicolumn{12}{|c|}{ Valor Pastoral de la pradera x Cobertura Arbórea } \\
\hline & & \multicolumn{3}{|c|}{$T 1: 0 \%$} & \multicolumn{3}{|c|}{ T2: $30 \%-40 \%$} & \multicolumn{3}{|c|}{ T3: $50 \%-60 \%$} & \multicolumn{3}{|c|}{$T 4:>70 \%$} \\
\hline & & 4.0 & \pm & 0.9 & 4.1 & \pm & 0.7 & 4.0 & \pm & 0.7 & 4.7 & \pm & 0.6 \\
\hline & 10 & 4.5 & \pm & 1.4 & 4.9 & \pm & 0.8 & 5.4 & \pm & 0.2 & 4.8 & \pm & 1.5 \\
\hline \multirow[t]{6}{*}{2014} & 11 & 4.9 & \pm & 2.1 & 6.3 & \pm & 0.7 & 5.4 & \pm & 0.5 & 4.8 & \pm & 0.9 \\
\hline & 12 & 4.6 & \pm & 2.1 & 4.8 & \pm & 0.3 & 3.4 & \pm & 1.4 & 3.5 & \pm & 2.0 \\
\hline & Total & 4.6 & \pm & $1.8 \mathrm{a}$ & 4.9 & \pm & $1.6 \mathrm{a}$ & 4.8 & \pm & $1.8 \mathrm{a}$ & 4.6 & \pm & $1.6 \mathrm{a}$ \\
\hline & 9 & 5.0 & \pm & 0.7 & 4.2 & \pm & 0.4 & 3.7 & \pm & 1.2 & 4.4 & \pm & 0.8 \\
\hline & 10 & 3.6 & \pm & 0.8 & 3.2 & \pm & 0.5 & 2.9 & \pm & 0.6 & 3.1 & \pm & 1.0 \\
\hline & 11 & 5.6 & \pm & 1.0 & 4.5 & \pm & 0.5 & 5.3 & \pm & 0.3 & 4.7 & \pm & 0.7 \\
\hline \multirow[t]{4}{*}{$2015-2016$} & 12 & 4.4 & \pm & 0.8 & 4.5 & \pm & 0.5 & 3.5 & \pm & 0.6 & 3.6 & \pm & 0.5 \\
\hline & 1 & 3.3 & \pm & 1.6 & 3.0 & \pm & 0.3 & 2.7 & \pm & 0.7 & 3.0 & \pm & 1.1 \\
\hline & 2 & - & & - & 0.9 & \pm & 1.6 & 0.8 & \pm & 1.4 & - & & - \\
\hline & Total & 4.9 & \pm & $2.2 \mathrm{a}$ & 4.0 & \pm & $1.6 \mathrm{a}$ & 3.9 & \pm & 1.9 a & 4.2 & \pm & $2.0 \mathrm{a}$ \\
\hline
\end{tabular}

Nota: Letras iguales indican sin diferencia estadísticamente significativa $(p<0.05)$.

TABLA 4. Resultado de Ancova entre variables microclimáticas, tratamientos y tiempo, utilizando las medidas de resumen promedio mensual para cada variable.

\begin{tabular}{|c|c|c|c|c|c|c|}
\hline \multirow{2}{*}{ Variable } & \multicolumn{2}{|c|}{ Tratamiento } & \multicolumn{2}{|c|}{ Tiempo (h) } & \multicolumn{2}{|c|}{ Tratamiento * Tiempo } \\
\hline & $F$ & Valor $p$ & $F$ & Valor $p$ & $F$ & Valor $p$ \\
\hline Temperatura Ambiente & 0.0168 & 0.9970 & 16.2444 & 0.0001 & 0.0057 & 0.9994 \\
\hline Humedad Relativa & 0.0121 & 0.9982 & 15.3473 & 0.0002 & 0.0021 & 0.9999 \\
\hline Humedad del Suelo & 0.0738 & 0.9739 & 0.2119 & 0.6465 & 0.0370 & 0.9904 \\
\hline Temperatura del suelo & 0.3016 & 0.8241 & 21.8494 & 0.0000 & 0.1794 & 0.9101 \\
\hline
\end{tabular}

F = valor calculado de distribución de probabilidad continua; $\mathrm{P}$ = Valor de probabilidad calculado. Nivel de significancia $\mathrm{p}<0.05$.

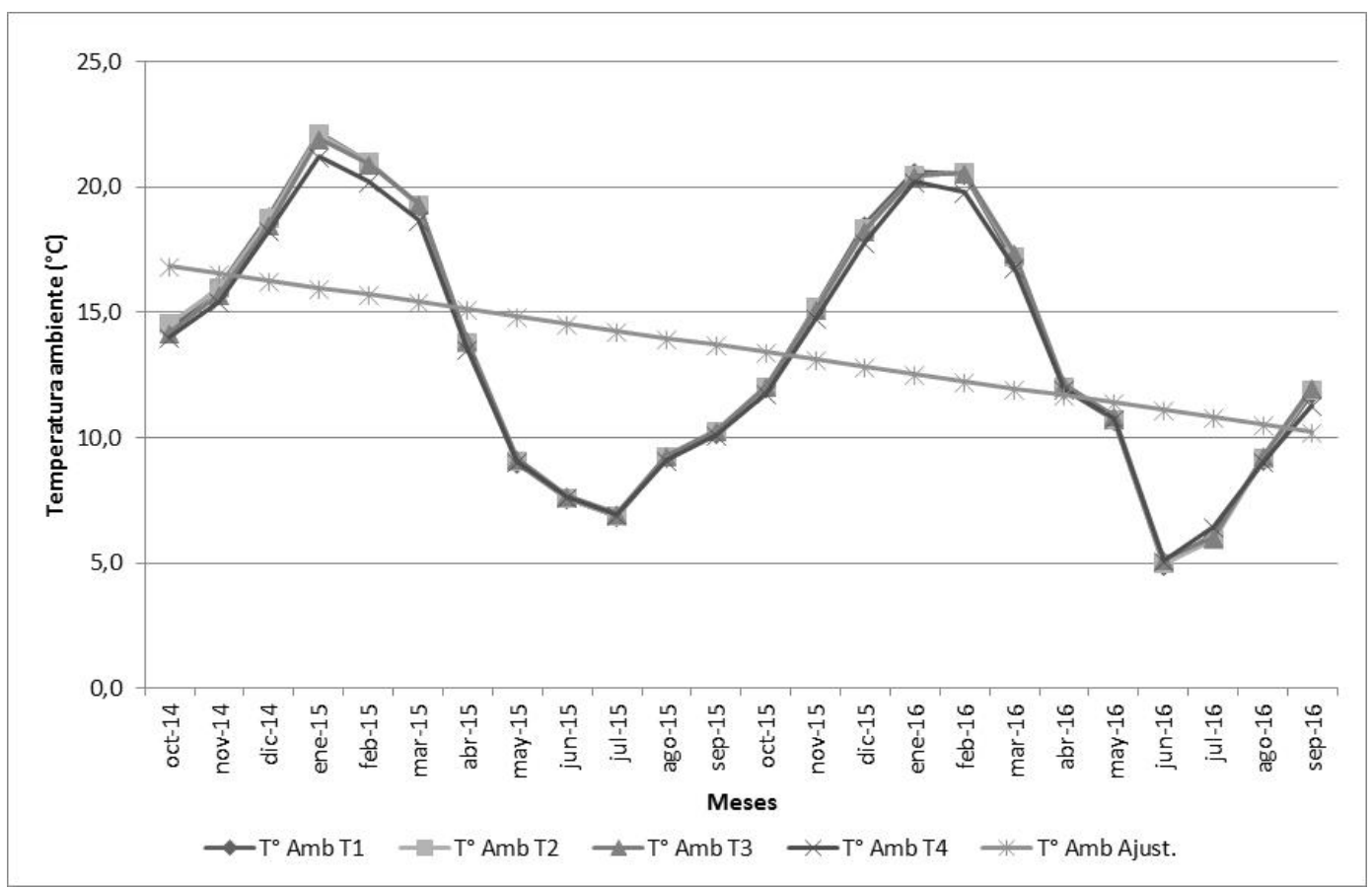

FIGURA 1. Comportamiento de temperatura ambiente por tratamiento en un periodo de 24 meses. $\mathrm{T} 1=0 \% 1 ; \mathrm{T} 2=30 \%$ - $40 \%$, T3 = 50\% - $60 \%$ y $\mathrm{T} 4=>70 \%$. 


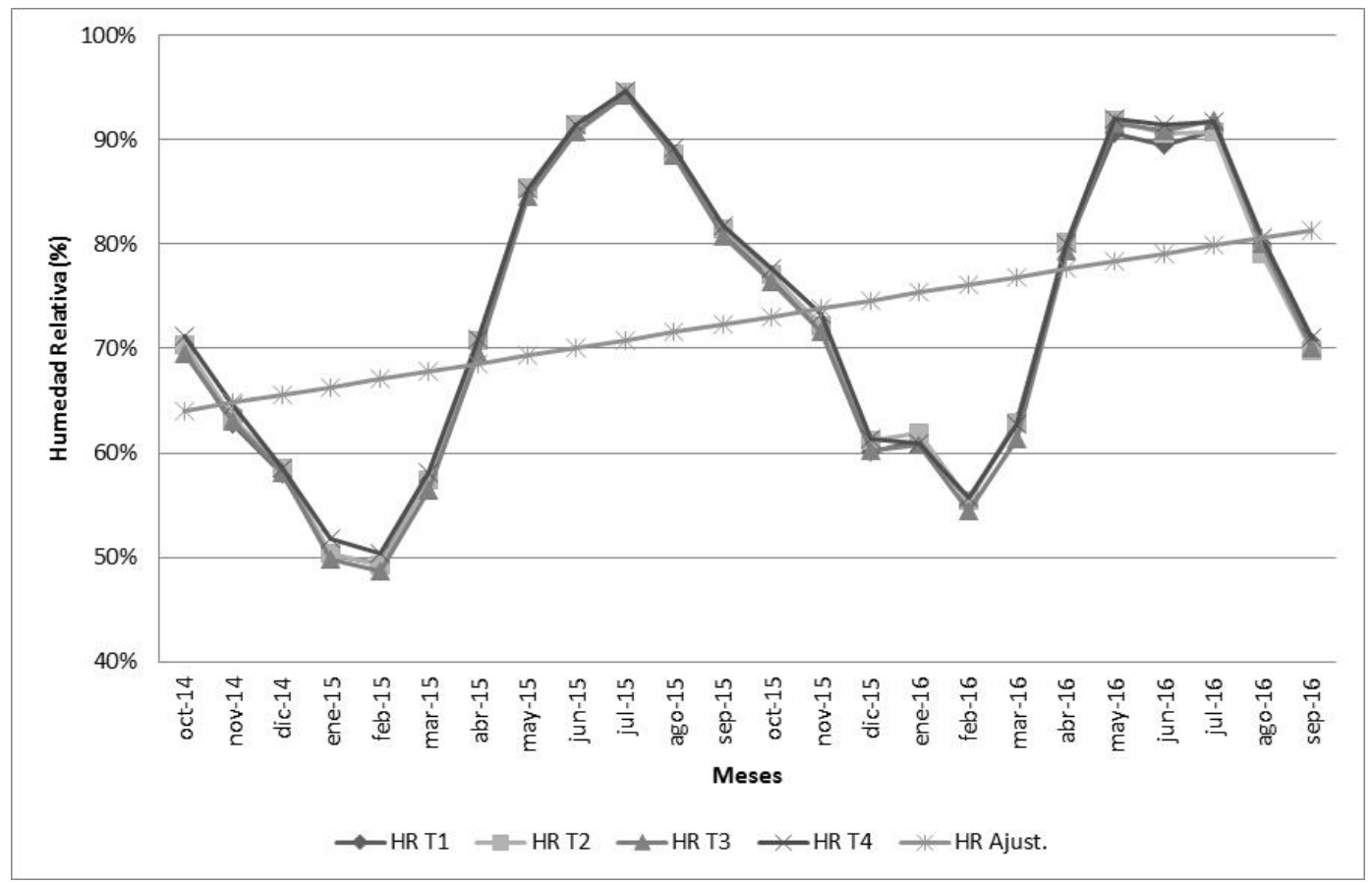

FIGURA 2. Comportamiento de la humedad relativa por tratamiento en un periodo de 24 meses. $\mathrm{T} 1=0 \% 1 ; \mathrm{T} 2=30 \%-40 \%, \mathrm{~T} 3=50 \%-60 \%$ y $\mathrm{T} 4=>70 \%$.

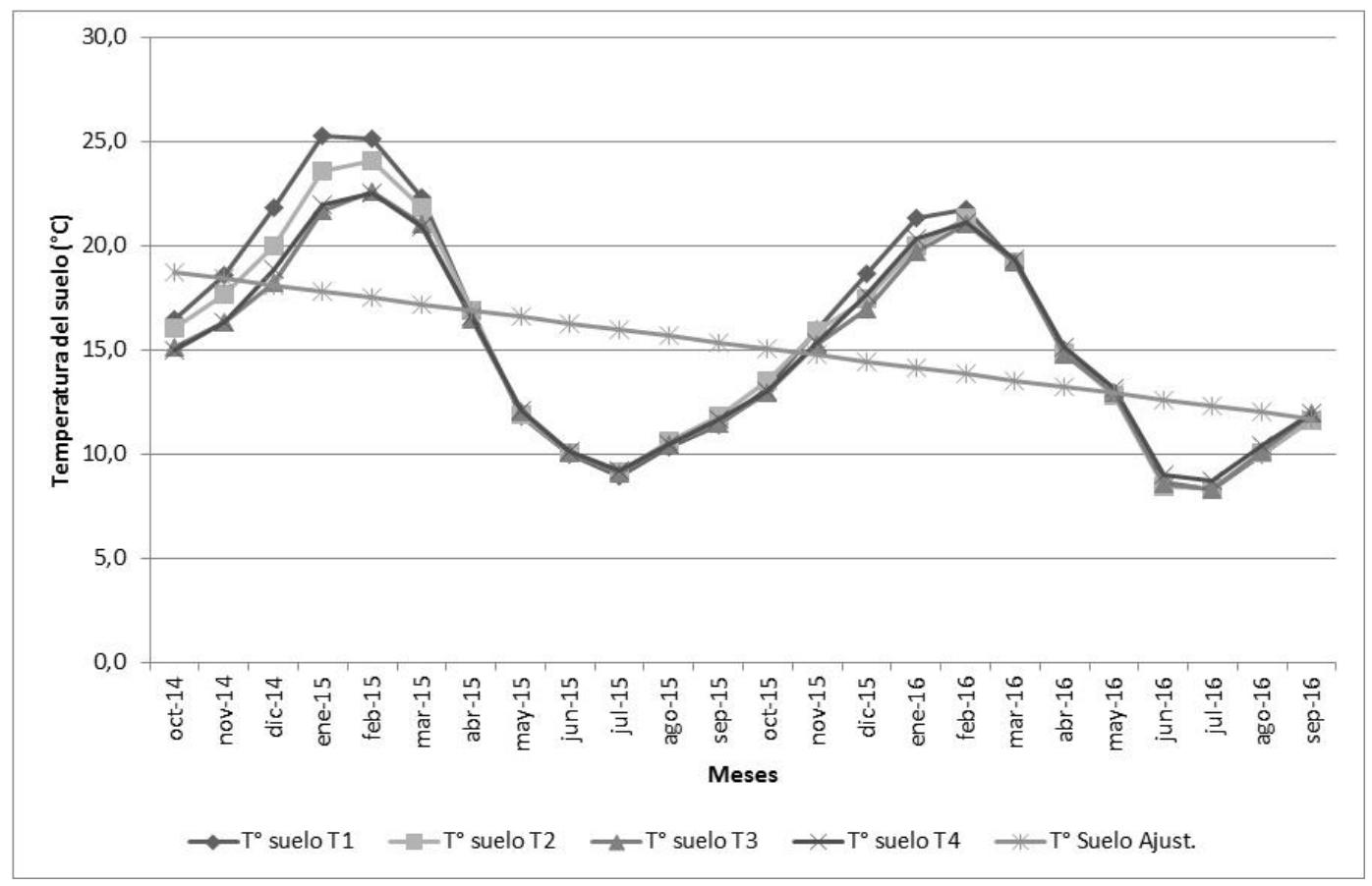

FIGURA 3. Comportamiento de temperatura del suelo por tratamiento en un periodo de 24 meses. $\mathrm{Tl}=0 \% 1 ; \mathrm{T} 2=30 \%-40 \%, \mathrm{~T} 3=50 \%$ - 60\% y $\mathrm{T} 4=>70 \%$. 


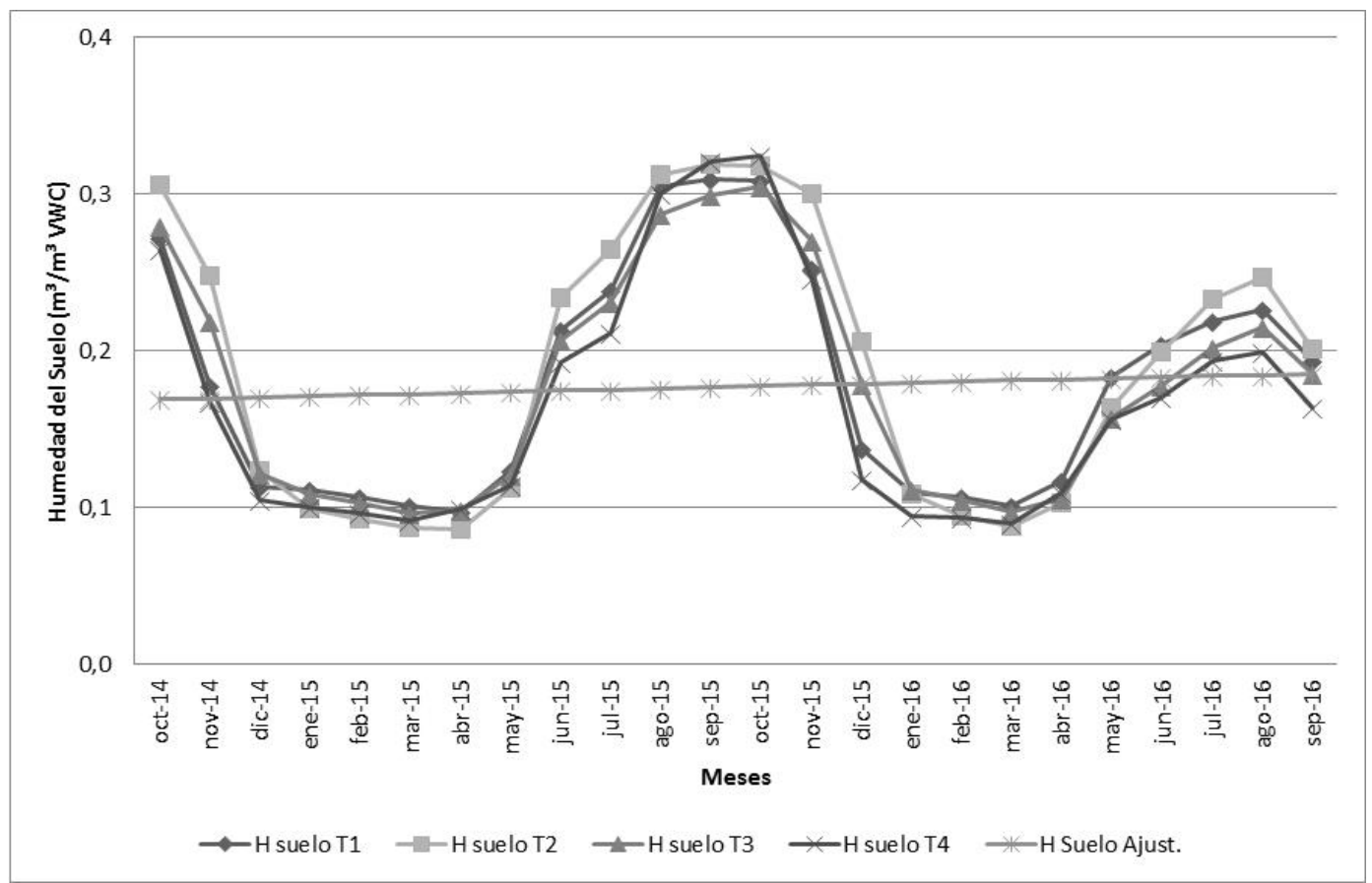

FIGURA 4. Comportamiento de humedad del suelo por tratamiento en un periodo de 24 meses. $\mathrm{Tl}=0 \% 1 ; \mathrm{T} 2=30 \%-40 \%, \mathrm{~T} 3=50 \%-60 \%$ y $\mathrm{T} 4=>70 \%$.

El tercer análisis también fue un modelo Ancova que consideraba como respuesta la productividad de la pradera, la cobertura arbórea como factor y las variables microclimáticas como covariables. Se debe especificar que para este análisis solo se consideraron los registros de las variables microclimáticas del mismo periodo en que se realizaron las mediciones de la productividad de la pradera para las dos temporadas de crecimiento, esto es, registros cada hora y para cada uno de los tratamientos y sus repeticiones de manera continua desde septiembre hasta diciembre del 2014, y desde septiembre del 2015 hasta febrero de 2016, correspondiendo a los meses en que hubo crecimiento de la pradera para cada temporada, con un total de más de 345000 datos. Los valores resumidos se muestran en la tabla 5 .

A través de este modelo se esperaba determinar el efecto de los diferentes tratamientos sobre variables microclimáticas, fue aplicado considerando diversos estadígrafos obtenidos a partir de la base completa de datos de los sensores establecidos en terreno y para ser representados como medidas de resumen. Para el análisis estadístico solo se utilizó el promedio mensual para los días completos o total (24 h), pues representa de mejor manera las respuestas de las variables microclimáticas, más que las máximas o mínimas o las de día o de noche. Lo anterior se constató al analizar previamente otras medidas de resumen como el valor máximo de cada variable microclimática (total, día y noche), el mínimo (total, día y noche) y el promedio (total, día y noche). En relación con estos antecedentes preliminares, el considerar en el análisis todas estas variables de manera conjunta incrementan sustancialmente la complejidad del modelo. Por ello y para efectos de este estudio, se decidió finalmente ajustar un modelo de regresión para cada variable microclimática de manera separada y para los tratamientos, construyéndose modelos diferenciados por tratamiento y además se consideraron cada una de las variables microclimáticas separadamente, y para valores mínimos, máximos y promedio. Los resultados de estos ajustes individuales se presentan en la tabla 6. 
TABLA 5. Variables microclimáticas promedio por año, mes y por tratamientos de cobertura arbórea.

\begin{tabular}{|c|c|c|c|c|c|c|c|c|c|c|c|c|c|c|c|c|c|c|c|c|c|c|c|c|}
\hline \multirow{2}{*}{ Año } & \multirow{2}{*}{ Mes } & \multicolumn{3}{|c|}{ T1: O\% } & \multicolumn{3}{|c|}{ T2: $30 \%-40 \%$} & \multicolumn{3}{|c|}{ T3: 50\% - 60\% } & \multicolumn{3}{|c|}{ T4: > 70\% } & \multicolumn{3}{|c|}{ Tl: O\% } & \multicolumn{2}{|c|}{ T2: $30 \%-40 \%$} & \multicolumn{3}{|c|}{ T3: 50\% - 60\% } & \multicolumn{3}{|c|}{ T4: > 70\% } \\
\hline & & \multicolumn{12}{|c|}{ Humedad relativa (\%) } & \multicolumn{11}{|c|}{ Temperatura ambiental $\left({ }^{\circ} \mathrm{C}\right)$} \\
\hline \multirow{3}{*}{2014} & 10 & 69.938 & \pm & 0.465 & 70.282 & \pm & 0.452 & 69.527 & \pm & 0.934 & 71.096 & \pm & 1.120 & 14.329 & \pm & 0.087 & 14.530 & \pm 0.279 & 14.224 & \pm & 0.043 & 14.002 & \pm & 0.363 \\
\hline & 11 & 62.781 & \pm & 0.774 & 63.352 & \pm & 1.454 & 63.015 & \pm & 1.431 & 64.547 & \pm & 1.357 & 16.037 & \pm & 0.138 & 15.939 & \pm 0.306 & 15.754 & \pm & 0.156 & 15.398 & \pm & 0.669 \\
\hline & 12 & 57.978 & \pm & 0.444 & 58.497 & \pm & 1.385 & 58.047 & \pm & 1.384 & 58.722 & \pm & 2.330 & 18.848 & \pm & 0.102 & 18.718 & \pm 0.347 & 18.509 & \pm & 0.219 & 18.232 & \pm & 0.921 \\
\hline \multirow{4}{*}{2015} & 9 & 81.309 & \pm & 0.806 & 81.485 & \pm & 0.617 & 80.773 & \pm & 0.825 & 81.757 & \pm & 1.005 & 10.195 & \pm & 0.115 & 10.207 & \pm 0.081 & 10.291 & \pm & 0.079 & 10.112 & \pm & 0.231 \\
\hline & 10 & 76.646 & \pm & 0.527 & 77.048 & \pm & 0.435 & 76.412 & \pm & 0.831 & 77.705 & \pm & 1.455 & 12.007 & \pm & 0.112 & 12.032 & \pm 0.062 & 12.056 & \pm & 0.102 & 11.793 & \pm & 0.394 \\
\hline & 11 & 71.742 & \pm & 0.269 & 72.164 & \pm & 0.359 & 71.633 & \pm & 0.630 & 73.314 & \pm & 2.509 & 15.231 & \pm & 0.033 & 15.211 & \pm 0.078 & 15.148 & \pm & 0.113 & 14.781 & \pm & 0.680 \\
\hline & 12 & 60.084 & \pm & 0.346 & 61.216 & \pm & 0.552 & 60.264 & \pm & 1.151 & 61.372 & \pm & 1.703 & 18.482 & \pm & 0.100 & 18.297 & \pm 0.170 & 18.263 & \pm & 0.212 & 17.802 & \pm & 0.864 \\
\hline \multirow{3}{*}{2016} & 1 & 61.188 & \pm & 0.270 & 61.949 & \pm & 0.409 & 60.776 & \pm & 0.664 & 61.269 & \pm & 4.103 & 20.616 & \pm & 0.048 & 20.460 & \pm 0.035 & 20.468 & \pm & 0.169 & 20.048 & \pm & 1.200 \\
\hline & 2 & 55.425 & \pm & 0.632 & 55.470 & \pm & 0.814 & 54.484 & \pm & 0.503 & 55.677 & \pm & 3.212 & 20.519 & \pm & 0.112 & 20.546 & \pm 0.046 & 20.571 & \pm & 0.151 & 19.877 & \pm & 1.151 \\
\hline & \multicolumn{13}{|c|}{ Humedad del suelo $\left(\mathrm{m}^{3} / \mathrm{m}^{3} \mathrm{VWC}\right)$} & \multicolumn{11}{|c|}{ Temperatura del suelo $\left({ }^{\circ} \mathrm{C}\right)$} \\
\hline \multirow{4}{*}{2014} & 10 & 0.272 & \pm & 0.043 & 0.306 & \pm & 0.018 & 0.279 & \pm & 0.042 & 0.265 & \pm & 0.042 & 16.481 & \pm & 1.016 & 16.070 & \pm 0.469 & 15.116 & \pm & 0.854 & 14.964 & \pm & 0.650 \\
\hline & 11 & 0.177 & \pm & 0.051 & 0.247 & \pm & 0.070 & 0.218 & \pm & 0.072 & 0.168 & \pm & 0.071 & 18.583 & \pm & 1.934 & 17.657 & \pm 0.997 & 16.295 & \pm & 0.639 & 16.329 & \pm & 0.862 \\
\hline & 12 & 0.113 & \pm & 0.020 & 0.123 & \pm & 0.059 & 0.122 & \pm & 0.029 & 0.104 & \pm & 0.062 & 21.793 & \pm & 2.022 & 19.985 & \pm 2.001 & 18.257 & \pm & 0.903 & 18.834 & \pm & 0.992 \\
\hline & 9 & 0.309 & \pm & 0.015 & 0.318 & \pm & 0.007 & 0.299 & \pm & 0.009 & 0.320 & \pm & 0.047 & 11.408 & \pm & 0.893 & 11.810 & \pm 0.544 & 11.463 & \pm & 0.081 & 11.678 & \pm & 0.508 \\
\hline \multirow{3}{*}{2015} & 10 & 0.309 & \pm & 0.019 & 0.318 & \pm & 0.004 & 0.305 & \pm & 0.020 & 0.325 & \pm & 0.038 & 13.159 & \pm & 0.936 & 13.517 & \pm 0.289 & 12.953 & \pm & 0.167 & 13.002 & \pm & 0.632 \\
\hline & 11 & 0.253 & \pm & 0.041 & 0.300 & \pm & 0.015 & 0.270 & \pm & 0.064 & 0.246 & \pm & 0.046 & 15.964 & \pm & 0.800 & 15.937 & \pm 0.484 & 15.253 & \pm & 0.036 & 15.345 & \pm & 0.450 \\
\hline & 12 & 0.138 & \pm & 0.039 & 0.205 & \pm & 0.079 & 0.178 & \pm & 0.068 & 0.118 & \pm & 0.034 & 18.637 & \pm & 0.492 & 17.469 & \pm 0.930 & 16.962 & \pm & 0.331 & 17.638 & \pm & 0.435 \\
\hline \multirow{2}{*}{2016} & 1 & 0.109 & \pm & 0.018 & 0.109 & \pm & 0.035 & 0.112 & \pm & 0.016 & 0.094 & \pm & 0.043 & 21.325 & \pm & 0.413 & 20.011 & \pm 0.949 & 19.703 & \pm & 0.652 & 20.325 & \pm & 0.633 \\
\hline & 2 & 0.107 & \pm & 0.020 & 0.094 & \pm & 0.027 & 0.105 & \pm & 0.018 & 0.093 & \pm & 0.044 & 21.790 & \pm & 0.789 & 21.350 & \pm 0.866 & 21.044 & \pm & 0.733 & 21.120 & \pm & 0.861 \\
\hline
\end{tabular}


TABLA 6. Coeficientes de determinación y significancia individual para efectos lineales $(\mathrm{X})$ y cuadráticos $\left(\mathrm{X}^{2}\right)$ en relación con la variable respuesta productividad de la pradera para cuatro variables microclimáticas en niveles mínimo, máximo y promedio y por tratamiento de cobertura arbórea.

\begin{tabular}{|c|c|c|c|c|c|c|c|c|c|c|c|c|}
\hline \multirow{3}{*}{$\begin{array}{l}\text { Variables } \\
\text { Microclimáticas }\end{array}$} & \multicolumn{3}{|c|}{$\mathrm{Tl}: \mathrm{O} \%$} & \multicolumn{3}{|c|}{$T 2: 30 \%-40 \%$} & \multicolumn{3}{|c|}{ T3: $50 \%-60 \%$} & \multicolumn{3}{|c|}{ T4: > 70\% } \\
\hline & \multirow{2}{*}{$R^{2}$} & \multicolumn{2}{|c|}{ valor $p$} & \multirow{2}{*}{$R^{2}$} & \multicolumn{2}{|c|}{ valor $p$} & \multirow{2}{*}{$R^{2}$} & \multicolumn{2}{|c|}{ valor $p$} & \multirow{2}{*}{$R^{2}$} & \multicolumn{2}{|c|}{ valor $p$} \\
\hline & & $x$ & $x^{2}$ & & $x$ & $x^{2}$ & & $x$ & $x^{2}$ & & $x$ & $x^{2}$ \\
\hline \multicolumn{13}{|c|}{ Humedad Relativa } \\
\hline Mínimo & 0.66 & $<0.0001$ & 0.0775 & 0.69 & $<0.0001$ & 0.3532 & 0.84 & $<0.0001$ & 0.0062 & 0.72 & $<0.0001$ & 0.0353 \\
\hline Máximo & 0.16 & 0.1253 & 0.1819 & 0.24 & 0.0116 & 0.7952 & 0.28 & 0.0094 & 0.2719 & 0.22 & 0.0144 & 0.9797 \\
\hline Promedio & 0.52 & $<0.0001$ & 0.1616 & 0.56 & $<0.0001$ & 0.2205 & 0.65 & $<0.0001$ & 0.1322 & 0.47 & 0.0002 & 0.3194 \\
\hline \multicolumn{13}{|c|}{ Humedad del suelo } \\
\hline Mínimo & 0.43 & 0.0003 & 0.5673 & 0.52 & $<0.0001$ & 0.7320 & 0.39 & 0.0009 & 0.3448 & 0.19 & 0.0294 & 0.7099 \\
\hline Máximo & 0.40 & 0.0009 & 0.2016 & 0.55 & $<0.0001$ & 0.2487 & 0.36 & 0.0012 & 0.6814 & 0.17 & 0.0353 & 0.8971 \\
\hline Promedio & 0.44 & 0.0003 & 0.2857 & 0.56 & $<0.0001$ & 0.3102 & 0.42 & 0.0003 & 0.6742 & 0.18 & 0.0321 & 0.8716 \\
\hline \multicolumn{13}{|c|}{ Temperatura Ambiente } \\
\hline Mínimo & 0.42 & 0.0014 & 0.0439 & 0.57 & $<0.0001$ & 0.6027 & 0.61 & $<0.0001$ & $\begin{array}{c}0.005 \\
6\end{array}$ & 0.53 & 0.0002 & 0.0114 \\
\hline Máximo & 0.40 & 0.0005 & 0.9249 & 0.56 & $<0.0001$ & 0.1086 & 0.56 & $<0.0001$ & 0.9938 & 0.49 & 0.0001 & 0.7768 \\
\hline Promedio & 0.51 & 0.0001 & 0.2278 & 0.67 & $<0.0001$ & 0.0804 & 0.71 & $<0.0001$ & 0.0527 & 0.55 & $<0.0001$ & 0.2512 \\
\hline \multicolumn{13}{|c|}{ Temperatura del suelo } \\
\hline Mínimo & 0.46 & 0.0003 & 0.1527 & 0.66 & $<0.0001$ & 0.9169 & 0.73 & $<0.0001$ & 0.0028 & 0.41 & 0.0008 & 0.1615 \\
\hline Máximo & 0.52 & 0.0001 & 0.0351 & 0.47 & 0.0001 & 0.7970 & 0.69 & $<0.0001$ & 0.0363 & 0.36 & 0.0014 & 0.4403 \\
\hline Promedio & 0.49 & 0.0002 & 0.1073 & 0.56 & $<0.0001$ & 0.7035 & 0.70 & $<0.0001$ & 0.0185 & 0.38 & 0.0012 & 0.2918 \\
\hline
\end{tabular}




\section{RESULTADOS}

En la tabla 2 se presentan los datos de productividad de la pradera $(\mathrm{PP})$ de manera mensual para cada tratamiento y por temporada. En la tabla 3 se muestran los valores pastorales (VP) para cada pradera al igual que en PP. La tabla 4 entrega los resultados del Ancova realizado para las variables microclimáticas asociados a los tratamientos, tiempo e interacción entre ambas. Las figuras 1, 2, 3 y 4 muestran para cada tratamiento, la variación de las distintas variables microclimáticas para un periodo completo de dos años.

Finalmente, la tabla 5 señala antecedentes de las variables microclimáticas como promedio por año, por mes y por tratamiento, y solo para los meses de cosecha de la pradera. La tabla 6 muestra los coeficientes de variación y significancia individual para efectos lineales $(\mathrm{x}) \mathrm{y}$ cuadráticos $\left(\mathrm{x}^{2}\right)$ en relación con la variable respuesta productividad de la pradera para cuatro variables microclimáticas en distintos niveles para cada tratamiento.

\section{DISCUSIÓN}

Efecto de la cobertura arbórea sobre la productividad de la pradera (PP) y valor pastoral (VP). PP no presentó diferencias significativas entre los tratamientos al primer año de establecido el ensayo. Esto se explicaría principalmente por la inercia del sistema productivo árbol-pradera, debido a décadas casi sin intervención alguna, solo la extracción menor y selectiva de árboles para la producción de carbón.

Para la segunda temporada, T1 $(0 \%$ de cobertura) mostró diferencias significativas en cuanto a generar una mayor PP que todos los otros tratamientos, los cuales entre sí no mostraron diferencias significativas (Tabla 1). Esta situación era de esperarse, dado que, desde el punto de vista de la naturaleza bioquímica de la energía ecológica contenida en el sistema, la producción orgánica se puede presentar en los ecosistemas solamente si los elementos químicos apropiados están disponibles en las concentraciones y cantidades adecuadas (Kimmins, 2004), lo cual puede ser aún constante en el sistema, independiente del tratamiento aplicado.

Además, de acuerdo con los datos de precipitación informados por la estación meteorológica del Instituto de Investigaciones Agropecuarias (INIA) Cauquenes, en la primera temporada evaluada, la precipitación y con ello la disponibilidad de humedad, fue menor que en la segunda temporada, lo cual resulta ser bastante relevante para la productividad de la pradera y podría explicar en parte esta diferencia (Ford et al., 2017). Por otro lado, es conocido que la capacidad sustentadora de un ecosistema es constante, aunque varíe el número y densidad de los estratos presentes (Peñaloza y Balocchi, 2002), y en la medida que disminuye o aumenta el tamaño y dominancia de un estrato, la o las otras aumentan o disminuyen proporcionalmente (Olivares, 1989). Todo esto podría explicar el hecho de que la pradera tenga una respuesta favorable inicial a la liberación a la que es sometida, producto de la ausencia total de la componente arbórea, aumentando sustancialmente su productividad, al existir condiciones de mayor luminosidad y con concentraciones de nutrientes, energía ecológica y agua disponible.

Situaciones similares fueron documentadas por Silva-Pando, González-Hernández y Rozados-Lorenzo (2002), en relación con la disponibilidad de luz. Estos autores determinaron que PP tiene un desarrollo importante a niveles de luminosidad sobre $70 \%$, que se puede asimilar a zonas sin presencia de árboles y, a su vez, este desarrollo se mantiene relativamente constante entre los niveles de $40 \%$ a $70 \%$, lo que se puede asociar con la cobertura arbórea de un sistema silvopastoril.

Sin embargo, estos resultados se contradicen con lo mencionado por Fernández et al. (2002) y Ovalle (1986), quienes indican que en general, a mayor cobertura de los árboles de espino, la productividad pratense también es mayor. Esto se explicaría principalmente por la condición de especie caducifolia leguminosa del espino que fija nitrógeno atmosférico, lo cual le confiere la particularidad de restituir constantemente los componentes órganominerales al ciclo de los nutrientes e incorporar este 
importante elemento $(\mathrm{N})$ al sistema. Por lo anterior, es esperable que los valores de productividad pratense, obtenidos inicialmente en este estudio, disminuyan con los años en relación directa con la disminución de la cobertura arbórea (Ovalle y Avendaño, 1984a; Fernández et al., 2002; Fernández, Lavín, Sotomayor, González y Tay, 2004).

Otro aspecto importante es que el espino tiene un desarrollo de las hojas desfasado de la pradera y una estructura de copa no muy densa Por esta razón, la competencia por luz es menor y se favorece el desarrollo de la pradera (Ovalle et al., 2015); situación que no ocurre con otras especies utilizadas en sistemas silvopastoriles. Lo anterior fue mencionado por Silva-Pando et al., (2002), quienes concluyeron que, en bosques de Pinus sp., existe una relación directamente proporcional entre la producción anual de las praderas y la transmisión de la luz a través del dosel arbóreo, lo que quiere decir que, al aumentar la disponibilidad de luz, también aumenta PP.

Por otro lado, en sistemas silvopastoriles con Pinus radiata (pino radiata), se ha indicado que el efecto de la cobertura sobre PP es indirectamente proporcional (Peñaloza y Balocchi, 2002), esto se debe a que, a diferencia del espino, el pino radiata es una especie de hoja perenne y de copa más densa, por lo que la competencia por luz en estos sistemas es más relevante que en los espinales, afectando la producción de pradera. Registros similares fueron señalados por Jose, Gillespie y Pallardy (2004), en ellos se menciona que la sombra provocada por los árboles de hojas perennes de sistemas agroforestales templados reduce los rendimientos de la componente herbácea, lo que.reforzaría la ventaja e importancia de la fenología característica del espino y su interacción positiva con la productividad pratense en un sistema silvopastoril.

El componente pratense no solamente es importante en un sistema silvopastoril en términos de la cantidad de materia seca que es capaz de producir, sino también en su calidad forrajera o valor pastoral (VP), que tiene directa relación con la composición botánica (CB) de la pradera. Respecto a la CB de la pradera (Tabla 3), se encontraron especies del tipo leguminosas (Medicago arabica; Medicago polymorpha), que en general presentan un valor pastoral alto a muy alto, del tipo gramíneas (Avena barbata; Briza spp.; Bromus mollis; Cynosorus echinatus; Danthonia spp.; Hordeum berteroanum; Lolium mutiflorum; Vulpia spp), con valores pastorales de medio a alto, y algunas juncáceas (Juncus spp.) y Liláceas (Linum spp.) ambas de escaso valor pastoral (Ovalle et al., 2015). Esta CB en cuanto a tipo y cantidad proporcional de especies presentes (Ecuación 2) determinan que, en general, la pradera tenga un VP promedio que fluctúa entre 3.9 y 4.9, situándola con un VP de bajo a medio para las praderas mediterráneas de Chile centro sur (Ovalle et al., 1981).

En cuanto al efecto de los tratamientos de cobertura y variables microclimáticas en el VP de la pradera, los resultados no muestran diferencias significativas en los distintos tratamientos de cobertura. Sin embargo, al analizar el VP en las diferentes etapas de desarrollo para todos los tratamientos, el mayor VP se registra durante los meses de octubre y noviembre para ambas temporadas de medición. En esas temporadas las variables microclimáticas muestran también mejores valores, que favorecen el crecimiento pratense; especialmente las variables humedad relativa y temperatura ambiente. Esto corrobora lo indicado por Moscoso y Bravo (2014), quienes señalan que la temperatura es uno de los factores principales que regula la tasa de crecimiento de la pradera.

Los datos de VP obtenidos no muestran diferencias entre los distintos tratamientos, a pesar de que otros autores han determinado que el VP bajo coberturas mayores de espino es mejor que en condiciones sin árboles, al

producirse en el tiempo una modificación de las especies pratenses, aumentando su VP en presencia del espino, dado principalmente por el remplazo de especies de menor VP por otras de mayor VP, producto de mejores condiciones edafoclimáticas a escala del micrositio (Ovalle y Avendaño, 1984a; Ramírez, 2011; Ovalle et al., 2015).

Estos trabajos indican que dicha variación se constató en periodos mayores de análisis a los contemplados en este estudio, debido a un proceso natural de adecuación de las especies herbáceas a las nuevas condiciones de 
luminosidad, temperatura, disponibilidad de agua y nutrientes, principalmente. Lo anterior, además de explicar estas diferencias, da claridad al hecho de que dos temporadas resultan ser insuficientes para determinar modificaciones del VP bajo diferentes coberturas. Por ello, se debe continuar con las mediciones de esta variable al menos dos temporadas más como mínimo y mantenerlas en el mediano y largo plazo.

\section{Efecto de la cobertura arbórea sobre las variables microclimáticas}

Este análisis presentó interacción estadísticamente significativa $(p<0.05)$, esto implica que las covariables afectan de diferente manera a las respuestas de PP, observándose tanto comportamientos lineales como cuadráticos para cada variable microclimática. Los análisis realizados indicaron que las cuatro variables microclimáticas no presentaban diferencias estadísticamente significativas respecto de la cobertura arbórea para dos años de mediciones. Se esperaba tener antecedentes que permitieran determinar los efectos de los distintos tratamientos en estas variables microclimáticas ya que, como se señaló anteriormente, la presencia de árboles genera sombra que atenúa el microclima (Ford et al., 2017), especialmente el térmico, mejorando los balances hídricos y tróficos de la pradera (Ovalle, 1986), así como la disponibilidad hídrica en el suelo, disminuyendo la evapotranspiración, reduciendo la temperatura del suelo y aumentando la humedad relativa, favoreciendo con ello la productividad herbácea (Ovalle y Avendaño, 1984b).

Para efectos de este estudio, si bien estos resultados se midieron adecuadamente a través de los sensores instalados (Tabla 5), estos no resultaron ser estadísticamente significativos, por lo que se debe seguir evaluando para observar si los tratamientos aplicados generan diferencias significativas. Por otro lado, para este análisis, solo la covariable tiempo resultó significativa, esta influyó significativamente en las variables microclimáticas de temperatura ambiente, humedad relativa y temperatura del suelo. La variable humedad del suelo no presentó diferencias significativas en el tiempo, en todos los meses evaluados para todos los tratamientos (Tabla 4).

A pesar de lo anterior, se pudieron observar comportamientos en las variables microclimáticas similares a las registrados por otros autores, asociados a la estepa de Acacia caven (Olivares et al., 1983; Ovalle y Avendaño, 1984b; Ovalle 1986; Ovalle et al., 2015). En este sentido, la humedad relativa resultó ser mayor en el tratamiento 4 de mayor cobertura, lo que puede asociarse con un efecto favorable por la presencia de árboles, en comparación con el tratamiento 1 sin árboles, que presenta los menores valores de HR. La humedad del suelo, en general, presentó valores relativamente constantes en todos los tratamientos, aunque los tratamientos con árboles presentaron mayores valores promedio, pero no significativamente distintos.

En relación con la temperatura ambiental, se observaron menores valores promedio en el tratamiento 4 $\left(15.8^{\circ} \mathrm{C}\right)$, siendo el de $0 \%$ de cobertura el que presentó mayores valores promedio $\left(16.3{ }^{\circ} \mathrm{C}\right)$. La temperatura del suelo presentó un comportamiento similar, pero con valores mayores, de entre $0.8^{\circ} \mathrm{C}$ y $1.5{ }^{\circ} \mathrm{C}$ promedio, para los tratamientos 4 y 1 , respectivamente. A pesar de lo anterior, aún no es posible apreciar si estas diferencias en variables microclimáticas generan un efecto significativo sobre VP y PP, situación que debiera seguir evaluándose en el largo plazo (Ford et al., 2017).

En las figuras 1, 2, 3 y 4, se puede observar que en aquellos tratamientos con presencia de árboles hay una tendencia a generar un efecto regulador en las diferentes variables microclimáticas, atenuando levemente las temperaturas máximas y mínimas, mejorando la humedad relativa y del suelo y principalmente entre los meses de octubre y noviembre, periodo en que la pradera presenta su mayor desarrollo (Moscoso y Bravo 2014). Lo anterior coincide con lo señalado por Ovalle y Avendaño (1984b) y Dube et al. (2013), quienes observaron que los árboles influyen positivamente en el crecimiento de la pradera, a través de la disminución de la temperatura del suelo y el aumento de la humedad relativa del aire, generando zonas 
más húmedas, creando un microclima que favorece el desarrollo de la pradera que crece bajo su influencia.

Respecto a la variable respuesta PP en relación con cada una de las variables microclimáticas, en la tabla 6 se puede apreciar que la variable microclimática que mejor explicaría la PP, es la bumedad relativa minima, la cual tiene efectos directamente proporcionales. Estos modelos presentaron un $\mathrm{R}^{2}$ de $0.66,0.69,0.84$ y 0.72 para los tratamientos 1, 2, 3 y 4, respectivamente. La segunda variable climática que también presenta buenos niveles de ajuste y que explicaría adecuadamente PP, es la temperatura ambiente promedio, la cual tiene efectos indirectamente proporcionales, con un $\mathrm{R}^{2}$ de $0.51,0.67,0.71$ y 0.55 para los tratamientos 1, 2, 3 y 4, respectivamente. Esto corrobora el hecho de que al disminuir la temperatura ambiente bajo cobertura en la temporada de primavera-verano, se genera una menor evapotranspiración en las plantas herbáceas, por lo que la humedad está más disponible para la pradera, favoreciendo su crecimiento (Ovalle y Avendaño, 1984b; Silva-Pando et al., 2002; Dube et al., 2013; Jose, Walter y Mohan, 2017).

\section{CONCLUSIONES}

Dos temporadas de crecimiento y de mediciones son insuficientes para observar efecto de los diferentes tratamientos aplicados sobre productividad de la pradera, valor pastoral, variables microclimáticas y sus interrelaciones. Sin embargo, y aunque no es significativamente diferente, las zonas arboladas (cobertura arbórea) presentan pequeñas diferencias en relación con las zonas no arboladas, generando variaciones leves en las variables microclimáticas, por lo que se puede esperar en el largo plazo contar con mejores condiciones que permitan un buen y adecuado desarrollo de la pradera que crece bajo la influencia arbórea que en sectores sin esa cobertura.

A pesar de lo esperado, los resultados de producción de la pradera indican mayor productividad estadísticamente significativa en el segundo año en la zona no arbolada (tratamiento 1), con valores de $3564.9 \mathrm{~kg} \mathrm{ha}{ }^{-1}$, en comparación con las zonas con árboles que presentaron en ese mismo periodo valores de $2088.9 \mathrm{~kg} \mathrm{ha}^{-1}, 1994.7 \mathrm{~kg} \mathrm{ha}$
1 y $1595.3 \mathrm{~kg} \mathrm{ha}^{-1}$ para los tratamientos 2, 3 y 4 respectivamente. Pero esto se asocia a una situación estacional que se explica por el efecto de liberación inicial de la pradera, que no es sostenible en el largo plazo; especialmente porque otros estudios han demostrado los efectos positivos que los árboles y, específicamente, una leguminosa como Acacia caven tiene en el ciclo de los nutrientes y en el crecimiento y distribución de las especies pratenses.

Finalmente, la etapa de caracterización del recurso arbóreo, a pesar de lo costoso que resulta esta actividad censal, facilitó la utilización de herramientas SIG que permitieron determinar la cobertura arbórea pre y post tratamientos y la posterior aplicación del Software ShadeMotion 3.0 para la determinación de sombras acumuladas en la unidades experimentales, dando mayor confiabilidad a la ubicación en terreno de la red de sensores de variables microclimáticas y mediciones de productividad pretense dentro de los parámetros de cobertura que se midieron para cada tratamiento. Sin embargo, y para fines operacionales o de fomento del manejo de los espinales bajo sistemas silvopastoriles, se hace necesario considerar en futuras investigaciones evaluar otras metodologías de caracterización, que permitan determinar principalmente la cobertura arbórea de manera más eficiente, que sean de fácil ejecución y de menor costo. En este sentido existen herramientas interesantes de evaluar, como podría ser el uso de fotografías hemisféricas y/o tecnologías más avanzadas como RPA o drones, evaluaciones que no fueron abordadas en este estudio.

\section{RECONOCIMIENTOS}

Los autores desean agradecer al Sr. Marco Antonio Bejar Vega, propietario del Predio "San Adolfo", por poner a disposición de esta investigación los espinales de su propiedad y dar todas las facilidades para que este estudio se desarrollara de acuerdo con lo programado. A la Comisión Nacional de Investigación Científica y Tecnológica de Chile (Conicyt), por su apoyo directo al autor principal de este estudio. Al Instituto Forestal de Chile Sede Biobío, por el apoyo entregado para el 
establecimiento, medición y posterior mantención de esta unidad de investigación, incorporándola de manera permanente en su Red de Unidades Demostrativas Agroforestales. A Jorge Acevedo, colaborador de Infor, por su apoyo técnico especializado en todas las actividades de terreno. Se agradece también a los investigadores del Instituto Forestal Sede Biobío, señores Juan Carlos Pinilla, Patricio Chung, Hernán Soto, Juan Carlos Muñoz y Alberto Ávila, por su apoyo profesional y técnico en diferentes etapas de este estudio.

\section{REFERENCIAS}

Cabello, A., \& Donoso, C. (2006). Acacia caven (Mol.) Mol. Las especies arbóreas de los bosques templados de Chile y Argentina. En C. Donoso (Ed.) Autoecología (pp. 126-134). Valdivia, Chile: María Cuneo Ediciones.

Castro, E. (2008). Manual de procedimientos para las estaciones meteorológicas. Sarapiquí. Costa Rica: Departamento Científico de La Selva y Manejo de Información. Organización para Estudios Tropicales.

Centro de Información de Recursos Naturales [Ciren] (1997). Estudio Agrológico VII Región. Descripciones de Suelos. Materiales y Símbolos. Publicación Ciren N¹17. Chile.

Di Castri, F., \& Hajek, E. (1976). Bioclimatología de Chile. Chile: Universidad Católica de Chile.

Dube, F., Thevathasan, N. V., Stolpe, N. B., Zagal, E., Gordon, A. M., Espinosa, M., \& Sáez, K. (2013). Selected C fluxes in Pinus ponderosa-based silvopastoral systems, exotic plantations and natural pastures on volcanic soil in the Chilean Patagonia. Agroforestry Systems 87(3), 527- 542. doi: 10.1007/s10457-012-9574-9

Fernández, F., Squella, F., \& Ovalle, C. (2002). Sistemas agroforestales: una alternativa de uso mixto de un mismo sitio con actividades silvoagropecuarias. En C. Pérez. Curso Internacional Manejo de microcuencas y prácticas conservacionistas de suelo y agua (pp. 95-118). Serie de Actas INIA N²2. Chillán Chile: INIA.

Fernández, F., Lavín, A., Sotomayor, J., González, M., \& Tay, J. (2004). Potencialidades de la agricultura y ganadería en el Secano Interior. En C. Ruiz S., C. Pérez C., \& K. Matsuya Sistemas productivos sustentables en el Secano Interior. Boletín Instituto de Investigaciones Agropecuarias [INIA] N¹25 (pp. 27-50). Chillán, Chile: INIA

Ford, M., Zamora, D., Current, D., Magner, J., Wyatt, G., Walter, W., \& Vaughan, S. (2017). Impact of managed woodland grazing on forage quantity, quality and livestock performance: the potential for silvopasture in Central Minnesota, USA. Agroforestry System, 93(1), 67-79. doi: 10.1007/s10457-017-0098-1

Jose, S., Gillespie, A., \& Pallardy, S. (2004). Interspecific interactions in temperate agroforestry. Agroforestry Systems, 61(1-3), 237-255. doi: 10.1023/B:AGFO.0000029002.85273.9b

Jose, S., Walter, D., \& Mohan K., B. (2017). Ecological considerations in sustainable silvopasture design and management. Agroforestry Systems, 19(1), 317-331. doi: 10.1007/s10457-016-0065-2

Kimmins, J. (2004). Biogeochemistry: Cycling of Nutrients in Ecosystems. In P. Benjamin-Cummings. Forest ecology: a foundation for sustainable forest management and environmental ethics in forestry (3a ed.) (pp. 71-129). Upper Saddle River, NJ, USA: Prentice Hall.

Moscoso, C., \& Bravo, R. (2014). Parámetros Climáticos y su efecto sobre las praderas. Agrisur, 70, 12-13.

Olivares, A., Cornejo, R., \& Gandara, J. (1983). Influencia de la estrata arbustiva (Acacia caven (Mol.)) Hook et Arn. en el crecimiento de la estrata herbácea. Avances en Producción Animal, 8(1-2), 19-28.

Olivares, A. (1989). El ecosistema silvopastoral. Avances en Producción Animal, 14(1-2), 3-14.

Olivares, A. (2006). Relaciones entre el estrato arbóreo, el estrato herbáceo y la conducta animal en el matorral de Acacia caven (espinal). Science et changements planétaires/Sécheresse 17(1), 333339.

Ovalle, C., Avendaño, J., Etienne, M., Muñoz, M., \& Serra, R. (1981). Determinación del valor pastoral en praderas naturales de la zona mediterránea subhúmeda. Agricultura Técnica (Chile), 4(4), 221-231.

Ovalle, C. (1986). Etude du système écologique sylvo-pastoral à Acacia Caven (Mol.) Hook. et Arn: aplications à la gestion des ressources renouvelables dans l'aire climatique méditerranéenne bumide et subbumide du Chile. Montpellier. France: INIA (Chile)- SubEstación Experimental de Cauquenes. Centre Emberger (Francia) Groupe d'Ecologie des Ressources Renouvelables.

Ovalle, C., \& Avendaño, J. (1984a). Utilización silvopastoral del espinal. I. Influencia del Espino (Acacia caven Mol.) sobre la productividad de la pradera natural. Agricultura Técnica (Chile), 44(4), 339-345.

Ovalle, C., \& Avendaño, J. (1984b). Utilización silvopastoral del espinal. II. Influencia del Espino (Acacia caven Mol.) sobre algunos elementos del medio. Agricultura Técnica (Chile), 44(4), 353-362. 
Ovalle, C., Casado, M., Acosta-Gallo, B., Castro, I., Del Pozo, A., Barahona, V., Sánchez-Jardón, L., De Miguel, J., Aravena, T., \& Martín-Forés, I. (2015). El espinal de la región mediterránea de Chile. Colección de Libros INIA N³4. Chile: Centro Regional de Investigación La Cruz-Instituto de Investigaciones Agropecuarias.

Ovalle, C., \& Squella, F. (1988). Terrenos de pastoreo con praderas anuales en el área de influencia climática mediterránea. En I. Ruiz (Ed.) Praderas para Chile (pp. 369409). Santiago. Chile: Instituto de Investigaciones Agropecuarias (INIA)-Ministerio de Agricultura.

Peñaloza, R., \& Balocchi, O. (2002). Componentes de los Sistemas Silvopastorales y sus interrelaciones. En N. Gaete (Ed.) Tecnologías apropiadas para la restauración ambiental integral de los suelos (pp.157-188). Serie Actas N²0. Carillanca. Chile: Instituto de Investigaciones Agropecuarias (INIA).

Quesada, F., Somarriba, E., \& Malek, M. (2005). ShadeMotion (versión 3.0) [Software de cómputo]. Costa Rica: Centro Agronómico Tropical de Investigación y Enseñanza (Catie) - Programa Agroambiental Mesoamericano.

Ramírez, R. (2011). Efecto de la sombra de Acacia caven (Mol.) Mol. en la pradera anual de clima mediterráneo. Tesis de licenciatura. Universidad de Chile, Santiago, Chile.

Santibáñez, F., \& Uribe, J. (1993). Atlas agroclimático de Chile: Regiones VI y VII. Santiago, Chile: Universidad de Chile.
Scholes, R., \& Archer, S. (1997). Tree-grass interactions in savannas. Annual Review of Ecology and Systematics, 28, 517544. doi: 10.1146/annurev.ecolsys.28.1.517

Silva-Pando, F., González-Hernández M., \& Rozados-Lorenzo, M. (2002). Pasture production in a silvopastoral system in relation with microclimate variables in the Atlantic coast of Spain. Agroforestry Systems, 56, 203-211. doi: 10.1023/A:1021359817311

Manuscrito recibido el 25 de junio de 2018

Aceptado el 12 de octubre de 2018

Publicado el 19 de agosto de 2019

Este documento se debe citar como:

Lucero I., A., Muñoz S., F., Cancino C., J., Sotomayor G., A., Dube, F., Sáez C., K., Villarroel M., A., \& Navarrete T., M. (2019). La cobertura arbórea de Acacia caven sobre la calidad de la pradera y microclima en un sistema silvopastoril de Chile central. Madera y Bosques, 25(2), e2521811. doi: 10.21829/myb.2019.2521811

Madera y Bosques por Instituto de Ecología, A.C. se distribuye bajo una Licencia Creative Commons Atribución-NoComercialCompartirlgual 4.0 Internacional. 